hep-ph/0003135

November 5, 2018

\title{
Power-Suppressed Thermal Effects from Heavy Particles
}

\author{
Eric Braaten and Yu Jia \\ Physics Department, Ohio State University, Columbus OH 43210, USA
}

\begin{abstract}
In quantum field theory, heavy particles with mass $M$ much greater than the temperature $T$ give not only effects suppressed by the Boltzmann factor $e^{-M / T}$, but also effects suppressed by powers of $T / M$. We show that power-suppressed terms in equilibrium observables arise from effective interactions among light particles due to virtual heavy particles. We study a model introduced by Matsumoto and Yoshimura in which heavy bosons interact only through a term that allows pair annihilation into light particles. We construct an effective Lagrangian for the light field by integrating out the heavy field, and use it to calculate the leading power-suppressed terms in the energy density. The thermal average of the Hamiltonian density for the heavy field includes a term proportional to $T^{6} / M^{2}$, but we show that this term can be eliminated by a field redefinition and therefore cannot have any physical significance.
\end{abstract}




\section{INTRODUCTION}

One might naively expect the effects of heavy particles with mass $M$ much greater than the temperature $T$ to be suppressed by the Boltzmann factor $e^{-M / T}$. However, in a quantum field theory, there are additional effects that are suppressed only by powers of $T / M$. In a recent series of papers [1] 34, Matsumoto and Yoshimura have argued that there are powersuppressed terms in the number density of heavy particles. If there were such terms, they could greatly exceed the contribution from the conventional Boltzmann-suppressed terms when $T \ll M$. This would have important implications for cosmology, because it would imply that the relic abundance of weakly-interacting massive particles is much larger than the conventional predictions based on the Boltzmann equation. Present bounds on the energy density of the universe would then imply significantly tighter constraints on the properties of the heavy particles that may constitute the cold dark matter.

Matsumoto and Yoshimura have studied the power-suppressed effects in a simple model with two species of scalar particles, one heavy and one light. The only interaction of the heavy particle is one that allows pair-annihilation into light particles. In the first two papers in the series [1], Matsumoto and Yoshimura used the influence functional method and a Hartree approximation to derive a quantum kinetic equation for the momentum distribution of heavy particles. Their equation includes off-shell effects associated with the thermal width of the heavy particles. They found that the leading power-suppressed contribution to the heavy-particle number density was proportional to $T^{7 / 2} / M^{1 / 2}$. In their third paper [2], Matsumoto and Yoshimura studied the equilibrium number density of heavy particles and found that the leading power-suppressed term was actually proportional to $T^{6} / M^{3}$. In their fourth paper [3], they derived a new quantum kinetic equation that reproduces the equilibrium result of Ref. [2].

Singh and Srednicki [4] have criticized Matsumoto and Yoshimura's conclusion that there are power-suppressed contributions to the number density of heavy particles. They argued that the quantum kinetic equation of Matsumoto and Yoshimura does not properly account for the interaction energy between the heavy particles and the thermal bath of light particles. They also noted that the power-suppressed terms found by Matsumoto and Yoshimura are actually contributions to the number density of virtual heavy particles. They argued that it is the number density of on-shell heavy particles that is relevant to the relic abundance, and this will have the usual Boltzmann suppression.

Srednicki recently argued that Matsumoto and Yoshimura's conclusion is the result of an inappropriate definition of the number density [5]. Srednicki considered a similar model in which the real-valued heavy field is replaced by a complex-valued field, so that the heavy bosons have a conserved charge. He showed that there was a definition of the heavy-particle number density that had Boltzmann suppression to all orders in perturbation

theory. Srednicki also suggested that the power-suppressed contributions to the heavyparticle energy density should have a simple interpretation in the effective field theory for the light particles obtained by integrating out the heavy particles. They are contributions to the energy density of light particles coming from nonrenormalizable effective interactions between the light particles.

The approach followed by Matsumoto and Yoshimura has been to integrate out the light field to get an quantum kinetic equation for the heavy particles. The power-suppressed terms 
are then understood as arising from the thermal width acquired by the heavy particle when the light field is integrated out. The heavy particle no longer has a sharp energy-momentum relation, but is a resonance. The power-suppressed terms come from the tail of the spectral function of the resonance, where the energy and momentum of the heavy particle are both small compared to $M$.

The philosophy of effective field theories [6] suggests using the diametrically opposite strategy. Physics involving energies and momenta small compared to $M$ can be understood most simply by integrating out the heavy field. A heavy particle whose energy and momentum is small compared to $M$ is off its mass-shell by an amount of order $M$. By the uncertainty principle, it can remain in this highly virtual state only for a time of order $1 / M$. Light fields can propagate only over distances of order $1 / M$ in this short time. Thus the effects of the highly virtual heavy particle on light fields with momenta much smaller than $M$ can be taken into account through local interactions among the light fields. In other words, the light fields can be described by a local effective field theory.

The effective field theory approach was used by Kong and Ravndal [7] to compute the leading power-suppressed terms in the energy density for QED at temperature $T \ll m_{e}$, where $m_{e}$ is the electron mass. They first integrated out the electron field to get a lowenergy effective Lagrangian for photons that includes the Euler-Heisenberg term. They then computed the energy density for this effective field theory at temperature $T$ and found that the leading term is proportional to $\alpha^{2} T^{8} / m_{e}^{4}$. This term can be identified as a contribution to the photon energy density coming from the Euler-Heisenberg term in the effective Hamiltonian for photons.

In this paper, we use similar effective field theory methods to study the power-suppressed thermal effects in the model considered by Matsumoto and Yoshimura. We show that the power-suppressed terms in the energy density can indeed be interpreted as contributions to the energy density of light particles from nonrenormalizable effective interactions. We also show that the term in the energy density from which Matsumoto and Yoshimura extracted the heavy-particle number density can be eliminated by a field redefinition and therefore can not have any physical significance.

This paper is organized as follows. We introduce the pair-annihilation model of Matsumoto and Yoshimura in section II and summarize their results on contributions to the energy density that are suppressed by powers of $T / M$. In section III, we construct an effective Lagrangian for the light field by integrating out the heavy field. We compute the leading power-suppressed terms in the energy density by differentiating the free energy density for the effective theory in equilibrium at temperature $T$. In section IV, we use a field redefinition to construct an effective Hamiltonian for the light field. We show that the thermal average of the effective Hamiltonian density reproduces the leading power-suppressed terms in the energy density. We summarize our results in section $\mathrm{V}$.

\section{BOSONIC MODEL WITH PAIR ANNIHILATION}

The model studied by Matsumoto and Yoshimura contains two species of spin-zero

particles: a heavy particle of mass $M$ described by the field $\varphi$ and a massless particle described by the field $\chi$. The Lagrangian density is 


$$
\mathcal{L}=\mathcal{L}_{\chi}+\frac{1}{2} \partial_{\mu} \varphi \partial^{\mu} \varphi-\frac{1}{2} M^{2} \varphi^{2}-\frac{1}{4} \lambda \varphi^{2} \chi^{2}
$$

where $\mathcal{L}_{\chi}$ is the Lagrangian for the light field:

$$
\mathcal{L}_{\chi}=\frac{1}{2} \partial_{\mu} \chi \partial^{\mu} \chi-\frac{1}{24} \lambda_{\chi} \chi^{4}
$$

We have suppressed the counterterms needed to remove ultraviolet divergences. The symmetry $\varphi \rightarrow-\varphi$ guarantees that the heavy particle is stable at zero temperature. The heavy particles can be created or annihilated in pairs via the $\varphi^{2} \chi^{2}$ interaction. Renormalizability also requires a $\varphi^{4}$ self-interaction, but we assume that its coefficient is much smaller than $\lambda$, so it can be neglected. This is a great simplification, because the Lagrangian is then quadratic in the heavy field $\varphi$. The $\chi^{4}$ term in (闻) is necessary for thermalization of the light field, but since we are primarily interested in the effects of the heavy field, we will carry out explicit calculations only to zeroth order in $\lambda_{\chi}$.

The energy density is the ensemble average of the Hamiltonion density: $\rho=\langle\mathcal{H}\rangle$. Matsumoto and Yoshimura divide the Hamiltonian density into three terms: $\mathcal{H}=\mathcal{H}_{\chi}+\mathcal{H}_{\varphi}+\mathcal{H}_{\text {int }}$, where

$$
\begin{aligned}
\mathcal{H}_{\chi} & =\frac{1}{2} \dot{\chi}^{2}+\frac{1}{2}(\nabla \chi)^{2}+\frac{1}{24} \lambda_{\chi} \chi^{4} \\
\mathcal{H}_{\varphi} & =\frac{1}{2} \dot{\varphi}^{2}+\frac{1}{2}(\nabla \varphi)^{2}+\frac{1}{2} M^{2} \varphi^{2} \\
\mathcal{H}_{\text {int }} & =\frac{1}{4} \lambda \varphi^{2} \chi^{2}
\end{aligned}
$$

We have suppressed the counterterms required to renormalize the composite operators so that their expectation values vanish at zero temperature. Matsumoto and Yoshimura interpreted the corresponding three terms in $\rho=\rho_{\chi}+\rho_{\varphi}+\rho_{\text {int }}$ as the energy density of the "thermal environment", the energy density of the "system" consisting of heavy particles, and the interaction energy density, respectively.

At zeroth order in $\lambda$, the energy density of the heavy particles is that of an ideal nonrelativistic gas,

$$
\rho_{\varphi}=M(M T / 2 \pi)^{3 / 2} e^{-M / T}
$$

which exhibits the usual Boltzman suppression. At second order in $\lambda$, there are terms that are suppressed only by powers of $T / M$. Matsumoto and Yoshimura calculated the leading power-suppressed contributions for each of the 3 terms in the energy density [2]:

$$
\begin{aligned}
\delta \rho_{\chi} & =-\frac{1}{69120} \lambda^{2} \frac{T^{6}}{M^{2}}, \\
\delta \rho_{\varphi} & =\frac{1}{69120} \lambda^{2} \frac{T^{6}}{M^{2}}, \\
\delta \rho_{\text {int }} & =-\frac{\pi^{2}}{64800} \lambda^{2} \frac{T^{8}}{M^{4}} .
\end{aligned}
$$

The terms proportional to $\lambda^{2} T^{6}$ terms cancel between $\delta \rho_{\chi}$ and $\delta \rho_{\varphi}$ and, so the leading power-suppressed term of order $\lambda^{2}$ in the total energy density is proportional to $\lambda^{2} T^{8}$. 
Matsumoto and Yoshimura noted this cancellation, but nevertheless interpreted $\delta \rho_{\varphi}$ in (8) as a contribution to the energy density of heavy particles. Taking the heavy particles to be nonrelativistic with energy equal to $M$, they identified $\delta \rho_{\varphi} / M$ as a contribution to the heavy-particle number density proportional to $T^{6} / M^{3}$.

Singh and Srednicki [4] have argued that the separation of the energy density into three terms corresponding to the system, the environment, and interactions is reasonable if and only if $\left|\rho_{\text {int }}\right| \ll \rho_{\varphi}$. If this condition is not satisfied, the coupling between the system and the environment is effectively strong and they cannot be clearly separated. Note that this condition is satisfied by the power-suppressed terms (8) and (9) if $T \ll M$.

\section{EFFECTIVE LAGRANGIAN}

In this section, we construct a low-energy effective Lagrangian for the light field $\chi$. This effective Lagrangian reproduces the zero-temperature Green functions at momentum scales much less than $M$. The effects of the heavy fields are reproduced by nonrenormalizable interactions with coefficients that are suppressed by powers of $1 / M^{2}$.

An effective Lagrangian for the light particles can be constructed by using functional methods to integrate out the heavy field. This method is particularly convenient for the model of Matsumoto and Yoshimura, because the Lagrangian is quadratic in the field $\varphi$. The effective action for the light field can be defined by a functional integral over the heavy field:

$$
\exp \left(i S_{\text {eff }}[\chi]\right) \equiv \int \mathcal{D} \varphi \exp \left(i \int d^{4} x \mathcal{L}\right)
$$

This effective action shouldn't be confused with the 1PI effective action that generates oneparticle-irreducible Green functions. Since $\mathcal{L}$ is quadratic in $\varphi$, the functional integral can be evaluated explicitly:

$$
S_{\mathrm{eff}}[\chi]=\int d^{4} x \mathcal{L}_{\chi}+\frac{i}{2} \ln \operatorname{det}\left(-\partial^{2}-M^{2}-\frac{\lambda}{2} \chi^{2}+i \epsilon\right) .
$$

The effective action can be expanded in powers of the coupling constant $\lambda$ :

$$
S_{\text {eff }}[\chi]=\int d^{4} x \mathcal{L}_{\chi}+\frac{i}{2} \ln \operatorname{det}\left(-\partial^{2}-M^{2}+i \epsilon\right)+\sum_{n=1}^{\infty} S_{\text {eff }}^{(n)}[\chi] .
$$

The term of $n$ 'th order in $\lambda$ is

$$
S_{\mathrm{eff}}^{(n)}[\chi]=-\frac{i \lambda^{n}}{2^{n+1} n} \operatorname{tr}\left[\left(-\partial^{2}-M^{2}+i \epsilon\right)^{-1} \chi^{2}\right]^{n} .
$$

The $\ln \operatorname{det}\left(-\partial^{2}-M^{2}\right)$ term in (12) can be discarded, because it is just a $\chi$-independent constant. The only contribution from $S_{\text {eff }}^{(1)}$ is the local functional $\int d^{4} x \chi^{2}$ with a divergent coefficient. It can be absorbed into the mass counterterm for the light field. The terms $S_{\text {eff }}^{(n)}$ for $n \geq 2$ are nonlocal functionals of the $\chi$ field. 
Since we are interested in light fields with characteristic momenta much smaller than $M$, we can use the derivative expansion to express $S_{\text {eff }}^{(n)}$ as an infinite series of local functionals. The derivative expansion is illustrated in Appendix A by expanding $S_{\text {eff }}^{(2)}$ to all orders. The lowest derivative term from $S_{\text {eff }}^{(2)}$ is a $\chi^{4}$ term. It has a divergent coefficient and can be absorbed into the counterterm for the $\chi^{4}$ term in the Lagrangian. The remaining terms have finite coefficients suppressed by powers of $1 / M^{2}$. They represent nonrenormalizable interactions among the light fields induced by virtual heavy particles. The effective action can now be expressed as the integral of an effective Lagrangian: $S_{\text {eff }}[\chi]=\int d^{4} x \mathcal{L}_{\text {eff }}$, where

$$
\mathcal{L}_{\text {eff }}=\mathcal{L}_{\chi}-\frac{\lambda^{2}}{96(4 \pi)^{2} M^{2}} \chi^{2} \partial^{2} \chi^{2}+\frac{\lambda^{2}}{960(4 \pi)^{2} M^{4}} \chi^{2}\left(\partial^{2}\right)^{2} \chi^{2}-\frac{\lambda^{3}}{96(4 \pi)^{2} M^{2}} \chi^{6}+\ldots
$$

We have suppressed the counterterms required to remove ultraviolet divergences, and we have kept all terms proportional to $\lambda^{m}\left(1 / M^{2}\right)^{n}$ with $m+n \leq 4$. If we consider an observable involving a single momentum scale $p \ll M$, terms in $\mathcal{L}_{\text {eff }}$ proportional to $\lambda^{m}\left(1 / M^{2}\right)^{n}$ will give effects suppressed by $\lambda^{m}(p / M)^{2 n}$. The $\chi^{6}$ term in (14) will therefore be comparable in importance to the $\chi^{2}\left(\partial^{2}\right)^{2} \chi^{2}$ term if $\lambda \sim(p / M)^{2}$.

A similar strategy can be used at nonzero temperature $T$ to compute power-suppressed contributions to equilibrium observables if $T \ll M$. Such observables can be expressed as Euclidean functional integrals over the fields $\varphi$ and $\chi$ with periodic boundary conditions in the Euclidean time direction. To calculate the power suppressed terms, we would first integrate over $\varphi$, then expand in powers of $\lambda$, then carry out the derivative expansion in powers of $1 / M^{2}$, and finally integrate over $\chi$. The first three steps reduce the problem to a calculation in an effective theory for $\chi$, and the final step of integrating over $\chi$ corresponds to computing the thermal average in that effective theory at temperature $T$. This strategy will reproduce all the terms that are suppressed by powers of $T / M$, but it will not give any Boltzmann-suppressed terms, because the expansion in powers of $1 / M^{2}$ eliminates terms with an essential singularity at $1 / M=0$.

The simplest way to compute the power-suppressed terms in the energy density $\rho$ is to calculate the corresponding terms in the free energy density $\mathcal{F}$ and then differentiate. The power-suppressed terms in $\mathcal{F}$ can be obtained simply by computing the free energy density at temperature $T$ for the theory defined by the effective Lagrangian (14). The leading terms are given by vacuum diagrams whose only vertex is one of the power-suppressed interactions in (14), and they can be written

$$
\begin{aligned}
\delta \mathcal{F}= & \frac{\lambda^{2}}{96(4 \pi)^{2} M^{2}}\left\langle\chi^{2} \partial^{2} \chi^{2}\right\rangle_{\text {free }}-\frac{\lambda^{2}}{960(4 \pi)^{2} M^{4}}\left\langle\chi^{2}\left(\partial^{2}\right)^{2} \chi^{2}\right\rangle_{\text {free }} \\
& +\frac{\lambda^{3}}{96(4 \pi)^{2} M^{2}}\left\langle\chi^{6}\right\rangle_{\text {free }}+\ldots,
\end{aligned}
$$

The angular brackets $\langle\ldots\rangle_{\text {free }}$ denote the thermal average in the free field theory. These thermal averages are expressed as Matsubara sum-integrals in Appendix B. The first term on the right hand side of (15) is zero. The remaining two terms give

$$
\delta \mathcal{F}=\frac{1}{1024}\left(\frac{16}{225} \lambda^{2} \frac{T^{4}}{M^{4}}-\frac{25}{48 \pi^{4}} \lambda^{3} \frac{T^{2}}{M^{2}}+\ldots\right) \mathcal{F}_{\text {free }} .
$$


where $\mathcal{F}_{\text {free }}=-\left(\pi^{2} / 90\right) T^{4}$ is the free energy of a gas of free massless bosons. By dimensional analysis, the $\chi^{2} \partial^{2} \chi^{2}$ term in (15) would have given a term proportional to $\lambda^{2} T^{6} / M^{2}$. The absence of such a term is related to the cancellation of the $\lambda^{2} T^{6} / M^{2}$ terms in the energy density noted by Matsumoto and Yoshimura.

Once the free energy density is known, we can derive the energy density by differentiation: $\rho=-T^{2} \frac{\partial}{\partial T}(\mathcal{F} / T)$. The leading power-suppressed terms in the energy density are

$$
\delta \rho=\frac{1}{1024}\left(\frac{112}{675} \lambda^{2} \frac{T^{4}}{M^{4}}-\frac{125}{144 \pi^{4}} \lambda^{3} \frac{T^{2}}{M^{2}}+\ldots\right) \rho_{\text {free }}
$$

where $\rho_{\text {free }}=\left(\pi^{2} / 30\right) T^{4}$ is the energy density of a free gas of massless bosons. Srednicki calculated the $\lambda^{2}(T / M)^{4}$ term for a similar model with a complex-valued heavy field by direct calculation in the full theory [5]. It is worth noting that the $\lambda^{3}(T / M)^{2}$ term is equally important if $\lambda \sim(T / M)^{2}$. At very low temperature, the power-suppressed terms in (17) dominate over the leading Boltzman-suppressed term (6) in the energy density of the heavy particles, but they represent small corrections to the energy density of the light particles.

\section{EFFECTIVE HAMILTONIAN}

Srednicki [5] argued that the power suppressed terms in the energy density should be interpreted as contributions to the energy density of the light field from nonrenormalizable effective interactions. In order to verify this explicitly, we construct a low-energy effective Hamiltonian density for the light field and compute its thermal average in the effective theory at temperature $T$.

If the Lagrangian density depends only on the field $\chi$ and its first derivatives, the standard Noether prescription for constructing the Hamiltonian density is $\mathcal{H}=\dot{\chi}(\partial \mathcal{L} / \partial \dot{\chi})-$ $\mathcal{L}$. This prescription can not be applied to the effective Lagrangian (14) because, even after using integration by parts to reduce the number of derivatives acting on any single field, it still depends on the second derivatives of $\chi$. While the Noether prescription for the Hamiltonian density can be generalized to higher derivative Lagrangians, it is rather cumbersome.

A simpler approach is to first construct a different effective Lagrangian $\mathcal{L}_{\text {eff }}^{\prime}$ that depends only on $\chi$ and its first derivatives, and then apply the Noether prescription to it. The effective Lagrangian $\mathcal{L}_{\text {eff }}$ in (14) is the unique effective Lagrangian that reproduces the off-shell Green's functions of the full theory at low momenta, but there are infinitely many effective Lagrangians that reproduce all the physical observables at low momenta. They include all effective Lagrangians that can be obtained from (14) by a field redefinition. In quantum field theory, we always have the freedom to redefine the field, because physical quantities, such as S-matrix elements, are invariant under field redefinitions. In renormalizable field theories, nontrivial field redefinitions are usually not considered, because they make the theory superficially nonrenormalizable. However, effective theories are already nonrenormalizable, so nontrivial field redefinitions don't introduce any additional complications. In fact they can be used to simplify the effective Lagrangian by removing terms that don't contribute to physical quantities. For example, by introducing the field redefinition $\chi \rightarrow \chi+G(\chi)$ into 
the kinetic term $\partial_{\mu} \chi \partial^{\mu} \chi$, we generate additional terms that can be used to cancel any terms of the form $G(\chi) \partial^{2} \chi$.

We use the following field redefinition to simplify the effective Lagrangian (14):

$$
\chi \longrightarrow \chi-\frac{\lambda^{2}}{72(4 \pi)^{2} M^{2}} \chi^{3}+\frac{\lambda^{2}}{720(4 \pi)^{2} M^{4}} \partial^{2} \chi^{3}+\ldots .
$$

Expanding out the derivatives and rearranging them by using integration by parts, our effective Lagrangian reduces to

$$
\mathcal{L}_{\text {eff }}^{\prime}=\mathcal{L}_{\chi}+\frac{\lambda^{2}}{240(4 \pi)^{2} M^{4}}\left(\partial_{\mu} \chi \partial^{\mu} \chi\right)^{2}-\frac{\lambda^{3}}{96(4 \pi)^{2} M^{2}} \chi^{6}+\ldots
$$

Again we have kept only those terms with a total of up to 4 powers of $\lambda$ and $1 / M^{2}$. This new effective Lagrangian will not reproduce the low-momentum Green functions of the original theory, but it will reproduce all physical observables involving low momenta.

An alternative way to derive the effective Lagrangian (19) starting from the original Lagrangian (11) is by matching physical quantities computed in both theories. We would begin by writing down the most general effective Lagrangian consistent with the symmetry $\chi \rightarrow-\chi:$

$$
\mathcal{L}_{\text {eff }}^{\prime}=\mathcal{L}_{\chi}+A \chi^{2} \partial_{\mu} \chi \partial^{\mu} \chi+B \chi^{6}+C\left(\partial_{\mu} \chi \partial^{\mu} \chi\right)^{2}+D \chi^{2} \partial^{2} \chi \partial^{2} \chi+E \chi \partial^{2} \chi \partial_{\mu} \chi \partial^{\mu} \chi+\ldots
$$

We would then write down the most general field redefinition consistent with the symmetry:

$$
\chi \rightarrow \chi+a \partial^{2} \chi+b \chi^{3}+c \chi \partial_{\mu} \chi \partial^{\mu} \chi+d \chi^{2} \partial^{2} \chi+\ldots
$$

Inserting this field redefinition into (20) and expanding it out, we would find that the coeffcients $b, c$, and $d$ could be used to set $A=D=E=0$. To determine the remaining coefficients, such as $B$ and $C$, we would exploit the fact that physical quantities are invariant under field redefinitions. We would compute $T$-matrix elements involving light particles with momenta $p \ll M$ in the full theory using the original Lagrangian (11) and in the effective theory using the effective Lagrangian (20). By matching these $T$-matrix elements, we would deduce that $B$ and $C$ have the values given in (19).

Having constructed the new effective Lagrangian $\mathcal{L}_{\text {eff }}^{\prime}$ in (19) that depends only upon $\chi$ and its first derivatives, we can use the standard Noether prescription to deduce the effective Hamiltonian. The effective Hamiltonian density reads $\mathcal{H}_{\text {eff }}=\mathcal{H}_{\chi}+\mathcal{H}_{\text {pow }}$, where $\mathcal{H}_{\chi}$ is given in (3) and $\mathcal{H}_{\text {pow }}$ includes all the higher dimension operators:

$$
\mathcal{H}_{\text {pow }}=\frac{\lambda^{2}}{240(4 \pi)^{2} M^{4}}\left(\partial_{\mu} \chi \partial^{\mu} \chi\right)\left(3 \dot{\chi}^{2}+(\nabla \chi)^{2}\right)+\frac{\lambda^{3}}{96(4 \pi)^{2} M^{2}} \chi^{6}+\ldots
$$

We can now calculate the power-suppressed terms in the energy density by taking the thermal average $\left\langle\mathcal{H}_{\text {eff }}\right\rangle$ at temperature $T$ for the effective theory defined by (19). The energy density can be written as $\rho=\left\langle\mathcal{H}_{\chi}\right\rangle+\left\langle\mathcal{H}_{\text {pow }}\right\rangle$. In $\left\langle\mathcal{H}_{\text {pow }}\right\rangle$, the leading power-suppressed terms $\delta \rho_{\text {pow }}$ are simply the thermal averages in a free field theory of the operators in (22). In $\left\langle\mathcal{H}_{\chi}\right\rangle$, 
the leading power-suppressed terms $\delta \rho_{\chi}$ come from treating the interactions in (19) as firstorder perturbations. The calculations are described in more detail in Appendix $\mathrm{B}$, and the results are

$$
\begin{aligned}
\delta \rho_{\chi} & =\frac{1}{1024}\left(\frac{16}{135} \lambda^{2} \frac{T^{4}}{M^{4}}-\frac{25}{24 \pi^{4}} \lambda^{3} \frac{T^{2}}{M^{2}}\right) \rho_{\text {free }} \\
\delta \rho_{\text {pow }} & =\frac{1}{1024}\left(\frac{32}{675} \lambda^{2} \frac{T^{4}}{M^{4}}+\frac{25}{144 \pi^{4}} \lambda^{3} \frac{T^{2}}{M^{2}}\right) \rho_{\text {free }}
\end{aligned}
$$

where $\rho_{\text {free }}=\left(\pi^{2} / 30\right) T^{4}$. The sum of (23) and (24) reproduces our previous result (17). Note that $\delta \rho_{\chi}$ in (23) differs from the power-suppressed terms in $\left\langle\mathcal{H}_{\chi}\right\rangle$ in the full theory, which include the term (7) suppressed by $\lambda^{2} T^{2} / M^{2}$.

If we had omitted the $\lambda^{2} \chi^{3}$ term in the field redefinition (18), the term proportional to $\chi^{2} \partial^{2} \chi^{2}$ in the effective Lagrangian (14) would not have been eliminated. There would then have been an additional term in the effective Hamiltonian proportional to $\chi^{2}\left(\dot{\chi}^{2}+(\nabla \chi)^{2}\right)$. Its thermal average reproduces the term $\delta \rho_{\varphi}$ in (8) calculated by Matsumoto and Yoshimura. However the $\chi^{2} \partial^{2} \chi^{2}$ interaction term in the effective Lagrangian gives an additional term in $\left\langle\mathcal{H}_{\chi}\right\rangle$ that reproduces $\delta \rho_{\chi}$ in (17). Since the cancelling contributions (7) and (8) to the energy density can be eliminated by a field redefinition, neither can have any physical significance. In particular, $\delta \rho_{\chi} / M$ cannot be interpreted as a contribution to the number density of heavy particles.

A field redefinition was also used by Kong and Ravandal [7] in their calculation of the energy density for QED at $T \ll m_{e}$. The effective Lagrangian obtained by integrating out the electron field is

$$
\mathcal{L}_{\text {eff }}=-\frac{1}{4} F_{\mu \nu} F^{\mu \nu}+\frac{\alpha}{60 \pi m_{e}^{2}} F_{\mu \nu} \partial^{2} F^{\mu \nu}+\frac{\alpha^{2}}{90 m_{e}^{4}}\left[\left(F_{\mu \nu} F^{\mu \nu}\right)^{2}+\frac{7}{4}\left(F_{\mu \nu} \tilde{F}^{\mu \nu}\right)^{2}\right]+\ldots
$$

The two power-suppressed terms are called the Uehling term and the Euler-Heisenberg term, respectively. The Uehling term can be eliminated by a field redefinition:

$$
A_{\mu} \longrightarrow A_{\mu}+\frac{\alpha}{30 \pi m_{e}^{2}} \partial^{2} A_{\mu}+\ldots
$$

It therefore cannot contribute to physical quantities. The leading power-suppressed term in the energy density comes from the Euler-Heisenberg interactions.

\section{CONCLUSION}

The effective-field-theory approach provides a simple way of understanding the contributions to equilibrium observables that are suppressed by powers of $T / M$. They arise from effective interactions among light particles that are induced by integrating out virtual heavy particles. The most economical way to compute the power-suppressed terms is to first construct a low-energy effective Lagrangian that describes the light particles at $T=0$ and then consider this effective theory in equilibrium at temperature $T$. 
For the pair annihilation model introduced in Refs. [1] [3], we demonstrated explicitly that the power-suppressed terms in the energy density can be interpreted as contributions from the light particles. We used the field redefinition (18) to construct an effective Hamiltonian density $\mathcal{H}_{\text {eff }}$ for the light field $\chi$, and then verified that its thermal average reproduces the power-suppressed terms. The field redefinition eliminated terms suppressed by $\lambda^{2}(T / M)^{2}$ from individual terms in $\left\langle\mathcal{H}_{\text {eff }}\right\rangle$, which otherwise would have canceled only after all such terms had been added together. The fact that all the $\lambda^{2}(T / M)^{2}$ terms can be eliminated by a field redefinition indicates that individual terms of this form cannot have any physical significance.

The incorrect conclusions concerning the heavy-particle number density in Ref. [2] stem from the authors having interpreted $\mathcal{H}_{\varphi}$ in (蛋) literally as an operator that creates only heavy particles and whose thermal average therefore probes the number density of those particles. However, the operator $\mathcal{H}_{\varphi}$ also creates light particles through loop diagrams that involve virtual heavy particles. Provided the momenta of the light particles are small compared to $M$, the loop diagram can be expressed as the product of a short-distance coefficient that depends on $M$ and a local effective operator that creates light particles. In the definition of the composite operator (4), the terms with effective operators $\chi^{2}, \dot{\chi}^{2},(\nabla \chi)^{2}$ and $\chi^{4}$ are implicitly subtracted, so $\mathcal{H}_{\varphi}$ creates light particles through higher dimension effective operators. Thus $\left\langle\mathcal{H}_{\varphi}\right\rangle$ receives contributions not only from heavy particles, but also from light particles created by these effective operators. It is these latter contributions that are responsible for the power-suppressed terms in the energy density. Those terms cannot be related to the number density of heavy particles that can participate in kinetic processes, since they involve only virtual heavy particles with lifetimes of order $1 / M$.

The strategy of effective field theory is to integrate out heavy fields to get an effective theory for light fields. The construction of a quantum kinetic equation for heavy particles requires exactly the opposite strategy. Light fields must be integrated out to create an effective description of the heavy particles. Effective field theory demonstrates convincingly that the quantum kinetic equations derived in Ref. [3] do not describe correctly the evolution of the number density of heavy particles. Perhaps the insights from effective field theory can be used as guidance for deriving the correct quantum kinetic equations.

\section{ACKNOWLEDGMENTS}

We thank A. Heckler and G. Steigman for bringing this problem to our attention. We thank J.O. Andersen for useful discussions. This work was supported in part by the U. S. Department of Energy Division of High Energy Physics (grant DE-FG02-91-ER40690).

\section{APPENDIX A: THE DERIVATIVE EXPANSION}

The derivative expansion can be used to express each of the term $S_{\text {eff }}^{(n)}$ in the effective action (12) as an infinite series of local functionals. In this appendix, we illustrate the

derivative expansion by applying it to the term $S_{\text {eff }}^{(2)}[\chi]$. The operator $\left(-\partial^{2}-M^{2}+i \epsilon\right)^{-1}$ in the definition (13) of $S_{\text {eff }}^{(n)}$ corresponds to the free spin-zero propagator: 


$$
G(x, y)=\int \frac{d^{4} q}{(2 \pi)^{4}} e^{-i q \cdot(x-y)} \frac{1}{q^{2}-M^{2}+i \epsilon} .
$$

The definition for $S_{\text {eff }}^{(2)}$ can be written

$$
S_{\text {eff }}^{(2)}[\chi]=-\frac{i \lambda^{2}}{16} \int d^{4} x \int d^{4} y G(x, y) \chi^{2}(y) G(y, x) \chi^{2}(x) .
$$

Inserting the integral expression (A.1) for the propagators, this becomes

$$
S_{\mathrm{eff}}^{(2)}[\chi]=-\frac{i \lambda^{2}}{16} \int d^{4} x \int d^{4} y \chi^{2}(x) \chi^{2}(y) \int \frac{d^{4} p}{(2 \pi)^{4}} e^{i p \cdot(x-y)} I\left(p^{2}\right),
$$

where the function $I\left(p^{2}\right)$ is given by

$$
I\left(p^{2}\right)=\int \frac{d^{4} q}{(2 \pi)^{4}} \frac{1}{q^{2}-M^{2}+i \epsilon} \frac{1}{(q+p)^{2}-M^{2}+i \epsilon} .
$$

This integral has a logarithmic ultraviolet divergence that can be isolated by adding and subtracting $I(0)$. The difference between the two integrals is convergent and can be evaluated using the Feynman parameter method:

$$
I\left(p^{2}\right)=I(0)-\frac{i}{(4 \pi)^{2}} \int_{0}^{1} d x \ln \left[1-x(1-x) p^{2} / M^{2}\right] .
$$

Assuming the integral in (A.3) is dominated by $p^{2} \ll M^{2}$, we can expand the logarithm into a power series in $p^{2}$ and then evaluate the Feynman parameter integral to get

$$
I\left(p^{2}\right)=I(0)+\frac{i}{(4 \pi)^{2}} \sum_{n=1}^{\infty} \frac{n !(n-1) !}{(2 n+1) !}\left(\frac{p^{2}}{M^{2}}\right)^{n} .
$$

The function $I\left(p^{2}\right)$ inside the integral over $p$ in (A.3) can be replaced by $I\left(-\partial_{x}^{2}\right)$ outside the integral. The integral over $p$ then reduces to $\delta^{4}(x-y)$, which collapses the expression to an integral over a single coordinate $x$. Our final result for the derivative expansion is

$$
S_{\text {eff }}^{(2)}[\chi]=-\frac{i \lambda^{2}}{16} I(0) \int d^{4} x \chi^{4}+\frac{\lambda^{2}}{16(4 \pi)^{2}} \sum_{n=1}^{\infty} \frac{(-1)^{n} n !(n-1) !}{(2 n+1) ! M^{2 n}} \int d^{4} x \chi^{2}\left(\partial^{2}\right)^{n} \chi^{2} .
$$

The $\chi^{4}$ term has a divergent coefficient, but it can be absorbed into one of the counterterms in the original Lagrangian. All the higher derivative terms have finite coefficients.

\section{APPENDIX B: THERMAL SUM-INTEGRALS}

The calculations of thermodynamic quantities in this paper can be reduced to computing thermal averages in a free field theory. In the imaginary-time formalism, these thermal averages are expressed as sums over Euclidean energies and integrals over spacial momentum. We use the following notation for these sum-integrals: 


$$
\sum_{P}=T \sum_{p_{4}} \int \frac{d^{3} p}{(2 \pi)^{3}} .
$$

The Euclidean 4-momentum is $P=\left(\mathbf{p}, p_{4}=2 \pi n T\right)$, where $n$ is any integer. We also use the notation $P^{2}=\mathbf{p}^{2}+p_{4}^{2}$.

Many of the sum-integrals required in this paper are thermal averages of local operators in a free field theory:

$$
\begin{aligned}
& \left\langle\chi^{2} \partial^{2} \chi^{2}\right\rangle_{\text {free }}=4 \psi_{P} \oiint_{Q} \frac{1}{P^{2}}, \\
& \left\langle\chi^{2}\left(\partial^{2}\right)^{2} \chi^{2}\right\rangle_{\text {free }}=4 \oiint_{P} \oiint_{Q} \frac{\left(P^{2}\right)^{2}+P^{2} Q^{2}+2(P \cdot Q)^{2}}{P^{2} Q^{2}}, \\
& \left\langle\chi^{6}\right\rangle_{\text {free }}=15\left(\xi_{P} \frac{1}{P^{2}}\right)^{3}, \\
& \left\langle\left(\partial_{\mu} \chi \partial^{\mu} \chi\right)^{2}\right\rangle_{\text {free }}=\oiint_{P} \xi_{Q} \frac{P^{2} Q^{2}+2(P \cdot Q)^{2}}{P^{2} Q^{2}}, \\
& \left\langle\left(\partial_{\mu} \chi \partial^{\mu} \chi\right)(\nabla \chi)^{2}\right\rangle_{\text {free }}=-\oint_{P} \oint_{Q} \frac{P^{2} \mathbf{q}^{2}+2 P \cdot Q \mathbf{p} \cdot \mathbf{q}}{P^{2} Q^{2}}, \\
& \left\langle\chi^{2}(\nabla \chi)^{2}\right\rangle_{\text {free }}=\oiint_{P} \oint_{Q} \frac{\mathbf{q}^{2}}{P^{2} Q^{2}} .
\end{aligned}
$$

We also need several sum-integrals that come from computing the thermal average of the free-field Hamiltonian density to first order in the effective interactions:

$$
\begin{aligned}
\left\langle\mathcal{H}_{\chi} i \int d^{4} x\left(\partial_{\mu} \chi \partial^{\mu} \chi\right)^{2}\right\rangle_{\text {free }} & =-2 \oint_{P} \oint_{Q} \frac{\left[P^{2} Q^{2}+2(P \cdot Q)^{2}\right]\left(P^{2}-2 \mathbf{p}^{2}\right)}{\left(P^{2}\right)^{2} Q^{2}}, \\
\left\langle\mathcal{H}_{\chi} i \int d^{4} x \chi^{6}\right\rangle_{\text {free }} & =-45\left(\sum_{Q} \frac{1}{Q^{2}}\right)^{2} \oiint_{P} \frac{P^{2}-2 \mathbf{p}^{2}}{\left(P^{2}\right)^{2}} \\
\left\langle\mathcal{H}_{\chi} i \int d^{4} x \chi^{2} \partial_{\mu} \chi \partial^{\mu} \chi\right\rangle_{\text {free }} & =\oint_{P} \oint_{Q} \frac{\left(P^{2}+Q^{2}\right)\left(P^{2}-2 \mathbf{p}^{2}\right)}{\left(P^{2}\right)^{2} Q^{2}} .
\end{aligned}
$$

We can simplify the sum integrals by averaging over angles using $\left\langle p^{i}\right\rangle=0$ and $\left\langle p^{i} p^{j}\right\rangle=$ $\mathbf{p}^{2} \delta^{i j} / 3$. This reduces the double sum-integrals to products of single sum-integrals. The single sum-integrals that are needed are

$$
\begin{gathered}
\xi_{P} P^{2}=0, \\
\oiint_{P} 1=0, \\
\sum_{P} \frac{1}{P^{2}}=\frac{1}{12} T^{2}, \\
\sum_{P} \frac{\mathbf{p}^{2}}{P^{2}}=\frac{\pi^{2}}{30} T^{4}, \\
\sum_{P} \frac{\mathbf{p}^{2}}{\left(P^{2}\right)^{2}}=\frac{1}{8} T^{2}, \\
\sum_{P} \frac{\left(\mathbf{p}^{2}\right)^{2}}{\left(P^{2}\right)^{2}}=\frac{\pi^{2}}{12} T^{4} .
\end{gathered}
$$


We have given only the temperature-dependent terms in the sum-integrals. The temperature-independent terms are ultraviolet divergent and depend on the choice of ultraviolet cutoff. The most convenient cutoff is dimensional regularization of the integrals over the spatial momenta. With this cutoff, the temperature-independent terms vanish. 


\section{REFERENCES}

[1] Sh. Matsumoto and M. Yoshimura, Relic Abundances due to Cosmic Pair Annihilation, hep-ph/9809326; Phys. Rev. D 59, 123511 (1999).

[2] Sh. Matsumoto and M. Yoshimura, Temperature Power Law of Equilibrium Heavy Particle Density, hep-ph/9910393.

[3] Sh. Matsumoto and M. Yoshimura, New Kinetic Equation for Pair-annihilating Particles: Generalization of the Boltzmann Equation, hep-ph/9910425.

[4] A. Singh and M. Srednicki, Phys. Rev. D 61, 023509 (2000).

[5] M. Srednicki, Thermal Abundances of Heavy Particles, hep-ph/0001090.

[6] See, for example, H. Georgi, Ann. Rev. Nucl. Part. Sci. 43, 209 (1993); D.B. Kaplan, Effective Field Theories, nucl-th/9506035; A.V. Manohar, Effective Field Theories, hepph/9606222; F. Ravndal, Applications of Effective Lagrangians, hep-ph/9708449.

[7] X. Kong and F. Ravndal, Nucl. Phys. B526, 627 (1998). 